\title{
Functional Organization of the Ventral Auditory Pathway
}

\author{
Yale E. Cohen, Sharath Bennur, Kate Christison-Lagay, Adam Gifford \\ and Joji Tsunada
}

\begin{abstract}
The fundamental problem in audition is determining the mechanisms required by the brain to transform an unlabelled mixture of auditory stimuli into coherent perceptual representations. This process is called auditory-scene analysis. The perceptual representations that result from auditory-scene analysis are formed through a complex interaction of perceptual grouping, attention, categorization and decision-making. Despite a great deal of scientific energy devoted to understanding these aspects of hearing, we still do not understand (1) how sound perception arises from neural activity and (2) the causal relationship between neural activity and sound perception. Here, we review the role of the "ventral" auditory pathway in sound perception. We hypothesize that, in the early parts of the auditory cortex, neural activity reflects the auditory properties of a stimulus. However, in latter parts of the auditory cortex, neurons encode the sensory evidence that forms an auditory decision and are causally involved in the decision process. Finally, in the prefrontal cortex, which receives input from the auditory cortex, neural activity reflects the actual perceptual decision. Together, these studies indicate that the ventral pathway contains hierarchical circuits that are specialized for auditory perception and scene analysis.
\end{abstract}

Keywords Auditory perception · Decision-making - Categorization - Perceptual grouping $\cdot$ Ventral auditory pathway

Y. E. Cohen $(\bowtie) \cdot$ S. Bennur $\cdot$ J. Tsunada

Department of Otorhinolaryngology, University of Pennsylvania, Philadelphia, USA

e-mail: ycohen@mail.med.upenn.edu

Y. E. Cohen

Department of Neuroscience, University of Pennsylvania, Philadelphia, USA

e-mail: ycohen@mail.med.upenn.edu

Department of Bioengineering, University of Pennsylvania, Philadelphia, USA

K. Christison-Lagay · A. M. Gifford

Neuroscience Graduate Group, University of Pennsylvania, Philadelphia, USA

(C) The Author(s) 2016

P. van Dijk et al. (eds.), Physiology, Psychoacoustics and Cognition in Normal and Impaired Hearing, Advances in Experimental Medicine and Biology 894,

DOI 10.1007/978-3-319-25474-6 40 


\section{Introduction}

Hearing and communication present a variety of challenges to the auditory system. To be heard and understood, the auditory brain must transform a time-varying acoustic stimulus into a perceptual representation; that is, a sound.

Auditory perception is associated with a number of computational processes, which may act in parallel or serial, including: perceptual grouping, decision-making, attention, and categorization. (1) Perceptual grouping is a form of feature-based stimulus segmentation that determines whether acoustic events will be grouped into a single sound or be segregated into distinct sounds (Bregman 1990). (2) Auditory decision-making is a computational process in which the brain interprets sensory information in order to detect, discriminate, or identify the source or content of auditory stimuli (Gold and Shadlen 2007). Did I hear the stimulus? From where and whom did it come? What does it tell me? How can I use this information to plan an action? (3) Although attention is not always necessary, our awareness of a sound can be influenced by attention (Alain and Arnott 2000; Micheyl et al. 2003; Fritz et al. 2005; Shinn-Cunningham 2008; Snyder et al. 2012; Gutschalk et al. 2015). For example, we can choose whether to listen to - or ignore - the first violin, the string section, or even the whole orchestra. Likewise, we can selectively attend to the particular features in a person's voice that allow a listener to identify the speaker. (4) In auditory categorization, sounds are classified based on their acoustic features or more processed forms of information (e.g., semantic knowledge), providing an efficient means to interact with stimuli in our environment (Ashby and Berretty 1997; Gifford et al. 2014). For example, when we hear the word "Groningen" from different speakers, we can categorize the gender of each speaker based on the pitch of the speaker's voice. On the other hand, to analyze the linguistic content transmitted by a speech sound, we can ignore the unique pitch, timbre etc. of each speaker and categorize the sound into the distinct word category "Groningen".

It is thought that the neural computations and processes that mediate auditory perception are found in the ventral auditory pathway (Rauschecker and Scott 2009; Romanski and Averbeck 2009; Hackett 2011; Bizley and Cohen 2013). In rhesus monkeys, this pathway begins in core auditory cortex - specifically, primary auditory cortex (A1) and the rostral field. These core areas project to the middle lateral $(\mathrm{ML})$ and anterolateral belt (AL) regions of auditory cortex. In turn, these belt regions project directly and indirectly to the ventrolateral prefrontal cortex (vlPFC).

It is important to briefly comment on the contribution of the dorsal ("spatial") pathway to auditory perception (Rauschecker 2012; Cloutman 2013). Spatial information can act as a grouping cue to assist the segregation of an acoustic stimulus into discrete sounds. For example, when a rhythmic sequence of identical sound bursts is presented from a single location, it is often perceived as one source. But, when the sound sequence is presented from two different locations, it can be perceived as two sounds (Middlebrooks and Onsan 2012; Middlebrooks and Bremen 2013). Such findings suggest that a mixture of spatial and non-spatial auditory cues from both the dorsal and ventral pathways may be needed in order to create a coherent auditory-perceptual representation that guides behavior. 
Nonetheless, in this review, we focus on the hierarchical processing that occurs at different stages in the ventral auditory pathway. In particular, we identify — or, at least, suggest - the unique contributions of these different processing stages to auditory perception and categorization, with an acknowledgment that associating any single brain region with a particular computation oversimplifies the complexity of the auditory brain. Indeed, it is well known that neurons become increasingly sensitive to more complex stimuli along the beginning stages of the ventral auditory pathway (e.g., between the core and belt regions of the auditory cortex). For example, core neurons are more sharply tuned for tone bursts than neurons in the lateral belt, whereas lateral-belt neurons are more selective for particular spectrotemporal features of complex sounds, such as vocalizations (Rauschecker and Tian 2000). Here, though, we review hierarchical processing in the ventral pathway by focusing on those studies in which neural activity was collected simultaneously while a listener was engaged in an auditory task.

\section{Neural Correlates of Auditory Perception Along the Ventral Auditory Pathway}

A1's role in auditory perception is controversial. Part of that controversy stems from the putative role of A1 in processing auditory "objects" (Nelken 2008). We will take the position that auditory objects are analogous to perceptual representations (i.e., sounds) (Bizley and Cohen 2013). As a consequence of this definition, if a neuron encodes an auditory object, it should be modulated by a listener's perceptual reports. That is, by holding a stimulus constant and testing whether neural activity is modulated by a listener's reports, neural activity that is associated with the acoustic features of the stimulus can be dissociated from neural activity associated with the perceptual report. Thus, neurons with complex tuning properties or even those modulated by components of a task (Brosch et al. 2005; Fritz et al. 2005) may contribute to the construction of a perceptual representation; but by themselves do not offer direct evidence of a perceptual representation.

There has been a recent set of literature implicating A1 in auditory perceptual decision-making (Riecke et al. 2009; Kilian-Hutten et al. 2011; Niwa et al. 2012; Riecke et al. 2012; Bizley et al. 2013). In one study, ferrets were asked to report changes in a sound's pitch, and it was found that both local-field potentials and spiking activity in A1 were modulated by the ferrets' pitch judgments. Niwa and colleagues have also shown that A1 single-unit activity is modulated by monkeys' reports during a task in which monkeys reported whether or not a sound was amplitude modulated. Finally, human-imaging studies have revealed that regions of core auditory cortex are modulated by listener's reports of the identity of an ambiguous speech sound. However, a different body of work suggests that A1 does not encode auditory decisions. For example, when monkeys discriminate between two types of acoustic flutter, A1 activity is not modulated by the monkeys' choices (Lemus et al. 2009b). 
What could be the bases for these apparent divergent sets of findings? We posit that these differences can be attributed to several non-exclusive possibilities. One possibility may be due to the relative perceptual and cognitive demands of the behavioural task: tasks with different demands might differentially engage neurons in core auditory cortex (Bizley and Cohen 2013; Nienborg and Cumming 2014). A second possibility focuses on how choice-related activity itself is analyzed. In choice analyses, it is imperative to restrict the analysis to those trials in which neural modulation related to choice can be clearly disassociated from the auditory stimulus. If this analysis is not carefully conducted, apparent choice activity may be conflated with stimulus-related activity. Finally, choice-related activity may not reflect a casual contribution of the auditory cortex to decision-making but may simply reflect feedback from higher choice-sensitive areas (Nienborg and Cumming 2009) or the structure of the correlated noise (Nienborg et al. 2012).

In the belt regions (ML and AL) of the ventral pathway, several lines of study from our laboratory suggest that this is not the case; but also see Niwa et al. 2013. While monkeys categorized speech sounds, we tested whether neural activity was modulated by monkeys' categorical judgements. We found that AL neurons were not modulated by the monkeys' reports (Tsunada et al. 2011, 2012). In a separate line of studies, we asked monkeys to listen to a sequence of tone bursts and report whether the sequence had a "low" or "high" pitch. We found that neither ML nor AL activity was modulated by the monkeys' choices (Tsunada et al., in press).

Do neurons in the different belt regions contribute differentially to auditory perception? The preliminary findings from our low-high pitch study suggest that AL neurons, but not ML neurons, might represent the sensory information (evidence) used to inform the monkeys' perceptual decisions. Specifically, AL neurometric sensitivity appeared to be correlated with both psychometric sensitivity and the monkeys' choices. Consistent with these findings, AL may play a causal role in these auditory judgments: microstimulation of an AL site tends to shift the monkeys' reports toward the pitch associated with the site's frequency tuning.

Whereas a single cortical locus of decision-making has yet to materialize, decision-related activity is seen throughout the frontal lobe. vlPFC neurons are strongly modulated by monkeys' choices (Russ et al. 2008; Lee et al. 2009). Neural activity in the inferior frontal lobe of the human cortex is also modulated by choice when listeners judge the content of ambiguous speech sounds (Binder et al. 2004). Neural correlates relating to a listeners' decision on auditory-flutter stimuli have also been observed in the ventral premotor cortex (Lemus et al. 2009a). Interestingly, as noted above, the dorsal pathway also contributes to auditory perception; consistent with that notion, activity in the human parietal lobe is modulated by listeners' choices (Cusack 2005).

In summary, we propose a model in which auditory information is hierarchically organized and processed in the ventral pathway. In early parts of the auditory cortex, neural activity encodes the acoustic features of an auditory stimulus and become increasingly sensitive to complex spectrotemporal properties (Rauschecker and Tian 2000). In later regions of the auditory cortex, this information informs perceptual judgments. However, neural activity that reflects a listener's perceptual judgments does not become apparent until the frontal lobe. 


\section{Category Representation in the Ventral Auditory Pathway}

Next, we review the manner in which auditory-category information is hierarchically organized in the ventral auditory pathway. In brief, we will highlight how feature-based categories are represented early; whereas in later parts of the pathway, we find representations of "abstract" categories, which combine acoustic information with mnemonic, emotional, and other information sources.

In core auditory cortex, neural activity codes the category membership of simple feature conjunctions. For example, categorical representations of frequency-contours have been identified (Ohl et al. 2001; Selezneva et al. 2006). These neurons encode the direction of a frequency contour (increasing or decreasing), independent of its specific frequency content. These categories may be found in the firing rates of individual neurons or may be observed as a result of population-level computations.

Categories for more complex stimuli, such as speech sounds and vocalizations, can be found in the lateral belt (Chang et al. 2010; Steinschneider et al. 2011; Tsunada et al. 2011; Steinschneider 2013). For example, AL neurons respond categorically, and in a manner consistent with listeners' behavioral reports, to morphed versions of two speech sounds ("bad" and "dad"). AL neurons also respond categorically to species-specific vocalizations; however, the degree to which AL (and listeners) can categorize these vocalizations is constrained by the vocalizations' acoustic variability (Christison-Lagay et al. 2014). In humans, the superior temporal gyrus is categorically and hierarchically organized by speech sounds (Binder et al. 2000; Chang et al. 2010; Leaver and Rauschecker 2010): phoneme categories are found in the middle aspect; word categories in the anterior-superior aspect; and phrases in the most anterior aspect (DeWitt and Rauschecker 2012; Rauschecker 2012).

Beyond the auditory cortex, neurons represent categories that are formed based on the abstract information that is transmitted by sounds. vlPFC neurons represent the valence of food-related calls (e.g., high quality food vs. low quality food) (Gifford et al. 2005). That is, vlPFC neurons encode the "referential" information that is transmitted by vocalizations, independent of differences in their acoustic properties. Prefrontal activity also contributes to the formation of categories that reflect the emotional valence of a speaker's voice (Fecteau et al. 2005) as well as the semantic information transmitted by multisensory stimuli (Joassin et al. 2011; Werner and Noppeney 2011; Hu et al. 2012). Together, these studies are consistent with the idea that ventral auditory pathway is an information-processing pathway that more complex stimuli and categories are processed in a hierarchically organized manner.

\section{Future Questions}

Of course, several fundamental questions remain. First, as alluded to above, understanding how feedforward versus feedback information contributes to neural correlates of perceptual judgments remains an open question. Second, the degree to 
which different types of auditory judgements differentially engage different components of the ventral pathway has yet to be fully articulated. A third question is to identify how the different computational processes (e.g., perceptual grouping, attention, decision-making, and categorization) that underlie auditory perception interact with one another. For example, it remains an open issue as to whether and how attention differentially modulates neural correlates of auditory perception at different hierarchical levels of the ventral auditory pathway (e.g., A1 versus AL) (Atiani et al. 2014). Another example is to identify the potential interactions between auditory perceptual grouping and decision-making. Finally, it is important to identify the manner by which the dorsal and ventral auditory pathways interact in order to form a consistent and coherent representation of the auditory scene.

Open Access This chapter is distributed under the terms of the Creative Commons AttributionNoncommercial 2.5 License (http://creativecommons.org/licenses/by-nc/2.5/) which permits any noncommercial use, distribution, and reproduction in any medium, provided the original author(s) and source are credited.

The images or other third party material in this chapter are included in the work's Creative Commons license, unless indicated otherwise in the credit line; if such material is not included in the work's Creative Commons license and the respective action is not permitted by statutory regulation, users will need to obtain permission from the license holder to duplicate, adapt or reproduce the material.

\section{References}

Alain C, Arnott SR (2000) Selectively attending to auditory objects. Front Biosci 5:d202-212

Ashby FG, Berretty PM (1997) Categorization as a special case of decision-making or choice. In: Marley AAJ (ed) Choice, decision, and measurement: essays in honor of R. Duncan Luce. Lawrence Erlbaum Associates, Hillsdale, pp 367-388

Atiani S, David SV, Elgueda D, Locastro M, Radtke-Schuller S, Shamma SA, Fritz JB (2014) Emergent selectivity for task-relevant stimuli in higher-order auditory cortex. Neuron 82(2):486-499. doi:10.1016/j.neuron.2014.02.029

Binder JR, Frost JA, Hammeke TA, Bellgowan PS, Springer JA, Kaufman JN, Possing ET (2000) Human temporal lobe activation by speech and nonspeech sounds. Cereb Cortex 10(5):512528

Binder JR, Liebenthal E, Possing ET, Medler DA, Ward BD (2004) Neural correlates of sensory and decision processes in auditory object identification. Nat Neurosci 7:295-301

Bizley JK, Cohen YE (2013) The what, where, and how of auditory-object perception. Nat Rev Neurosci 14:693-707

Bizley JK, Walker KM, Nodal FR, King AJ, Schnupp JW (2013) Auditory cortex represents both pitch judgments and the corresponding acoustic cues. Curr Biol 23(7):620-625 (Research Support, Non-U.S. Gov't)

Bregman, AS (1990) Auditory scene analysis. MIT Press, Boston, MA

Brosch M, Selezneva E, Scheich H (2005) Nonauditory events of a behavioral procedure activate auditory cortex of highly trained monkeys. J Neurosci 25(29):6797-6806

Chang EF, Rieger JW, Johnson K, Berger MS, Barbaro NM, Knight RT (2010) Categorical speech representation in human superior temporal gyrus. Nat Neurosci 13:1428-1432

Christison-Lagay KL, Bennur S, Lee JH, Blackwell J, Schröder T, Cohen YE (2014) Natural variability in species-specific vocalizations constrains behavior and neural activity. Hear Res 312:128-142 
Cloutman LL (2013) Interaction between dorsal and ventral processing streams: where, when and how? Brain Lang 127(2):251-263. doi:10.1016/j.bandl.2012.08.003

Cusack R (2005) The intraparietal sulcus and perceptual organization. J Cogn Neurosci 17:641651

DeWitt I, Rauschecker JP (2012) Phoneme and word recognition in the auditory ventral stream. Proc Natl Acad Sci U S A 109(8):E505-E514

Fecteau S, Armony JL, Joanette Y, Belin P (2005) Sensitivity to voice in human prefrontal cortex. J Neurophysiol 94(3):2251-2254. doi:10.1152/jn.00329.2005

Fritz J, Elhilali M, Shamma S (2005) Active listening: task-dependent plasticity of spectrotemporal receptive fields in primary auditory cortex. Hear Res 206(1-2):159-176. doi: S03785955(05)00083-3 [pii] 10.1016/j.heares.2005.01.015

Gifford GW, 3rd, MacLean KA, Hauser MD, Cohen YE (2005) The neurophysiology of functionally meaningful categories: macaque ventrolateral prefrontal cortex plays a critical role in spontaneous categorization of species-specific vocalizations. J Cogn Neurosci 17(9):1471-1482

Gifford AM, Cohen YE, Stocker AA (2014) Characterizing the impact of category uncertainty on human auditory categorization behavior. PloS Comp Biol 10:e1003715

Gold JI, Shadlen MN (2007) The neural basis of decision making. Annu Rev Neurosci 30:535-574

Gutschalk A, Rupp A, Dykstra AR (2015) Interaction of streaming and attention in human auditory cortex. PLoS One 10(3):e0118962

Hackett TA (2011) Information flow in the auditory cortical network. Hear Res 271:133-146

Hu Z, Zhang R, Zhang Q, Liu Q, Li H (2012) Neural correlates of audiovisual integration of semantic category information. Brain Lang 121(1):70-75. doi:10.1016/j.bandl.2012.01.002

Joassin F, Maurage P, Campanella S (2011) The neural network sustaining the crossmodal processing of human gender from faces and voices: an fMRI study. Neuroimage 54(2):1654-1661. doi:10.1016/j.neuroimage.2010.08.073

Kilian-Hutten N, Valente G, Vroomen J, Formisano E (2011) Auditory cortex encodes the perceptual interpretation of ambiguous sound. J Neurosci 31(5):1715-1720. doi:10.1523/jneurosci.4572-10.2011

Leaver AM, Rauschecker JP (2010) Cortical representation of natural complex sounds: effects of acoustic features and auditory object category. J Neurosci 30:7604-7612

Lee JH, Russ BE, Orr LE, Cohen YE (2009) Prefrontal activity predicts monkeys' decisions during an auditory category task. Fron Integr Neurosci, 3(16)

Lemus L, Hernandez A, Romo R (2009a) Neural encoding of auditory discrimination in ventral premotor cortex. Proc Natl Acad Sci U S A 106(34):14640-14645. doi:10.1073/pnas.0907505106

Lemus L, Hernandez A, Romo R (2009b) Neural codes for perceptual discrimination of acoustic flutter in the primate auditory cortex. Proc Natl Acad Sci U S A 106:9471-9476

Micheyl C, Carlyon RP, Shtyrov Y, Hauk O, Dodson T, Pullvermuller F (2003) The neurophysiological basis of the auditory continuity illusion: a mismatch negativity study. J Cogn Neurosci 15(5):747-758. doi:10.1162/089892903322307456

Middlebrooks JC, Bremen P (2013) Spatial stream segregation by auditory cortical neurons. J Neurosci 33:10986-11001

Middlebrooks JC, Onsan ZA (2012) Stream segregation with high spatial acuity. J Acoust Soc Am 132:3896-3911

Nelken I (2008) Processing of complex sounds in the auditory system. Curr Opin Neurobiol $18: 413-417$

Nienborg H, Cumming BG (2009) Decision-related activity in sensory neurons reflects more than a neuron's causal effect. Nature 459(7243):89-92. doi:10.1038/nature07821

Nienborg H, Cumming BG (2014) Decision-related activity in sensory neurons may depend on the columnar architecture of cerebral cortex. J Neurosci 34(10):3579-3585. doi:10.1523/jneurosci.2340-13.2014

Nienborg H, Cohen MR, Cumming BG (2012) Decision-related activity in sensory neurons: correlations among neurons and with behavior. Annu Rev Neurosci 35:463-483 (Review)

Niwa M, Johnson JS, O’Connor KN, Sutter ML (2012) Active engagement improves primary auditory cortical neurons' ability to discriminate temporal modulation. J Neurosci 32(27):93239334 
Niwa M, Johnson JS, O'Connor KN, Sutter ML (2013) Differences between primary auditory cortex and auditory belt related to encoding and choice for AM sounds. J Neurosci 33(19):83788395

Ohl FW, Scheich H, Freeman WJ (2001) Change in pattern of ongoing cortical activity with auditory category learning. Nature 412(6848):733-736

Rauschecker JP (2012) Ventral and dorsal streams in the evolution of speech and language. Front Evol Neurosci 4:7

Rauschecker JP, Scott SK (2009) Maps and streams in the auditory cortex: nonhuman primates illuminate human speech processing. Nat Neurosci 12(6):718-724

Rauschecker JP, Tian B (2000) Mechanisms and streams for processing of "what" and "where" in auditory cortex. Proc Natl Acad Sci U S A 97(22):11800-11806

Riecke L, Mendelsohn D, Schreiner C, Formisano E (2009) The continuity illusion adapts to the auditory scene. Hear Res 247(1):71-77. doi:10.1016/j.heares.2008.10.006

Riecke L, Vanbussel M, Hausfeld L, Baskent D, Formisano E, Esposito F (2012) Hearing an illusory vowel in noise: suppression of auditory cortical activity. J Neurosci 32(23):8024-8034. doi:10.1523/jneurosci.0440-12.2012

Romanski LM, Averbeck BB (2009) The primate cortical auditory system and neural representation of conspecific vocalizations. Annu Rev Neurosci 32:315-346

Russ BE, Orr LE, Cohen YE (2008) Prefrontal neurons predict choices during an auditory samedifferent task. Curr Biol 18(19):1483-1488

Selezneva E, Scheich H, Brosch M (2006) Dual time scales for categorical decision making in auditory cortex. Curr Biol 16(24):2428-2433

Shinn-Cunningham BG (2008) Object-based auditory and visual attention. Trends Cogn Sci 12:182-186

Snyder JS, Gregg MK, Weintraub DM, Alain C (2012) Attention, awareness, and the perception of auditory scenes. Front Psychol 3:15

Steinschneider M (2013) Phonemic representations and categories. In Cohen YE, Popper AN, Fay RR (eds), Neural correlates of auditory cognition, vol 45. Springer-Verlag, New York, pp 151-161

Steinschneider M, Nourski KV, Kawasaki H, Oya H, Brugge JF, Howard MA (2011) Intracranial study of speech-elicited activity on the human posterolateral superior temporal gyrus. Cereb Cortex 10:2332-2347

Tsunada J, Lee JH, Cohen YE (2011) Representation of speech categories in the primate auditory cortex. J Neurophysiol 105:2634-2646

Tsunada J, Lee JH, Cohen YE (2012) Differential representation of auditory categories between cell classes in primate auditory cortex. J Physiol 590:3129-3139

Tsunada, J, Liu, AS, Gold, JI, Cohen, YE ( ? ) Causal role of primate auditory cortex in auditory perceptual decision-making. Nature neuroscience (in press)

Werner S, Noppeney U (2011) The contributions of transient and sustained response codes to audiovisual integration. Cereb Cortex 21(4):920-931. doi:10.1093/cercor/bhq161 